\title{
SEGURANÇA EMOCIONAL COMO NECESSIDADE HUMANA BĀSICA - ATUAÇĀO DO ENFERMEIRO JUNTO AO CLIENTE EM UNIDADE DE TERAPIA INTENSIVA
}

\author{
Teresinha de Jesus Monteiro Espírito Santo ${ }^{1}$
}
ESPÍRITO SANTO, T. J. M. Segurança emocional como necessidade humana básica - atuação do enfermeiro junto ao cliente em unidade de terapia intensiva. Rev. Bras. Enf., Brasília, 38(3/4): 231-236, jul./d ez. 1985.

RESUMO. Pesquisa descritiva exploratória que se propõe a investigar a atuação do enfermeiro da Unidade de Terapia Intensiva, referenciando como este identifica, diagnostica e resolve os problemas ligados à segurança emocional dos clientes internados. $O$ estudo abrangeu vinte e oito enfermeiros e dez clientes das unidades de três hospitais da Cidade do Rio de Janeiro. A técnica adotada para pesquisa consistiu da observação sistemótica não participada. Os resultados obtidos demonstraram que os procedimentos terapêuticos dominantes recaem sobre alterações físicas em detrimento do emocional, evidenciando que o enfermeiro, ao distanciar-se de uma atuação holística, acarretará sérios prejuízos na assistência destes clientes, cuja necessidade humana básica afetada é predominantemente relacionada à segurança emocional.

ABSTRACT. This descriptive explanatory research proposes to search the performance of the persons who work at the Intensive Therapy Unit, referring to the way they identify, diagnose and solve the problems related to the emotional assurance of the inward patient. The study comprehended twenty-eight nurses and ten patients from the unities of three hospitals in Rio de Janeiro City. The technic which was used to search, consisted of the observation. The obtained results showed that the predominant therapeutic behavior indicate the physical alteration in detriment of the emotional, making evident that when the person who takes care of the patients leaves the whole performance, it will cause serious damages to the help for these patien ts whose basic human necessity affected is predominately related to the emotional assurance.

\section{INTRODUÇÃO}

Da preocupação com o aperfeiçoamento da assistência de saúde a pacientes graves, surgiram, em Hospitais da América Latina nas duas últimas décadas e principalmente no Brasil, as Unidades de Tratamento Intensivo (UTI).

As Unidades de Tratamento Intensivo foram criadas de forma a atender pacientes graves de modo permanente e exclusivista, sem acasionar os transtornos que certamente acarretariam, caso es- tes estivessem em enfermarias. $O$ prejuízo da não centralização destes clientes em um núcleo especializado seria sentido não somente por eles, mas também pelos pacientes de menor gravidade, que seriam naturalmente privados da assistência às suas necessidades em face das necessidades mais urgentes dos clientes de maior gravidade. Contribuiu ainda para a criação destes núcleos, além da proposta de melhor qualidade de individualidade da assistência a pacientes graves, os problemas de ordem econômico-administrativa, pois seria necessá-

\footnotetext{
1 Prof essor Assistente do Centro de Ciências Biológicas e da Saúde - Curso de Enfermagem - UNI-RIO.
} 
rio um número bem maior de profissionais para o atendimento destes clientes em clínicas comuns, sendo mais lógica a centralização de pessoal em uma única unidade que dispusesse de recursos hu. manos e materiais, associados a uma infra-estrutura que permitisse o seu funcionamento quase como um micro-hospital, dentro de uma estrutura hospitalar.

Foi portanto obedecendo aos "princípios de cuidados progressivos ao paciente", recomendados pela OPS ${ }^{16}$, quando esta considera os hospitais como estabelecimentos para o desenvolvimento de uma "Assistência Progressiva ao Paciente", que a UTI foi criada. Atualmente esta se constitui em seu principal núcleo, uma vez que seu princípio fundamental é de, através da utilização racional e objetiva dos recursos existentes, melhorar a qualidade da atenção à saúde, mediante a concentração de recursos altamente especializados, capazes de reduzir a morbidade e mortalidade.

Se lembrarmos, porém, que os clientes em UTI encontram-se acometidos de desequilíbrios orgânicos graves, poder-se-ia afirmar que sua segurança emocional, fatalmente, estará seriamente afetada, visto que, sendo o homem um ser holístico, a doença representa um fator capaz de afetar a segurança física, econômica, religiosa e, principalmente, emocional. Esta, conforme menciona SILVA ${ }^{20}$, relacionada com todas as necessidades humanas básicas, exige do enfermeiro maior atenção no que se refere às manifestações de insegurança dos clientes que se encontram na UTI.

Portanto, ao reconhecer-se que a segurança emocional como necessidade humana básica encontra-se seriamente afetada em clientes na UTI, pode-se estimar a importância do enfermeiro como profissional desta unidade pois, ao prestar assistência contínua nas 24 horas, é ele o elemento mais indicado à identificação de sinais e sintomas que expressem desequilíbrios emocionais, sendo ainda capaz de intervir de modo a apoiar e promover o ajustamento do cliente ao meio, contribuindo efetivamente para o retorno a uma situação de equilíbrio.

\section{SITUAÇÃO, PROBLEMA E SUA IMPORTÂNCIA}

Adotando-se a Teoria Holística, proposta por GEORGE \& LEVINE $^{8}$, temos que o homem é um todo dinâmico, onde todas as funções orgânicas são integradas, quer na saúde ou na doença.

Baseando-se neste pressuposto, pode-se afir- mar que a identificação de problemas referentes à necessidade humana básica de segurança emocional envolve as características pessoais relaciona. das à personalidade tanto do ser cliente como do ser enfermeiro. Tais características influenciam o desenvolvimento da assistência de enfermagem, sen do exigido do enfermeiro uma percepção ampla e cientificamente fundamentada acerca dos sinais e sintomas que expressam insegurança emocionais do cliente.

A percepção de problemas pelo enfermeiro na UTI é fortemente influenciada por suas vivências, conhecimentos, valores éticos e pessoais. Pode-se mesmo afirmar que são estes elementos associados os norteadores da conduta, ao se prestar assistência. Tais elementos por si só não são suficientes, sendo imprescindível que, ao se estabelecer um relacionamento terapêutico, esteja-se consciente da complexidade que envolve o ser humano.

A importância do enfermeiro da UTI na humanização da assistência aos clientes que se encontram sob seus cuidados, adquire uma maior proporção uma vez que, ao aproximar-se do cliente como ser humano que é, e ao estabelecer uma inter-relação efetiva, poderá identificar as formas pelas quais o cliente manifesta sua insegurança emocional. Conseqüentemente, será capaz de propor uma assistência de forma que, ao ajudar na superação deste desequilíbrio, permitirá o atendimento à necessidade afetada visando a individualidade do cliente como algo a ser preservado.

\section{OBJETIVOS}

Considerando-se a segurança emocional como necessidade humana básica, traçaram-se os seguintes objetivos para este estudo:

- Identificar os problemas mais comuns relativos à necessidade de segurança emocional em clientes na UTI;

- Verificar de que modo são identificados e diagnosticados pelo enfermeiro da UTI os probls:mas afetos à segurança emocional;

- Identificar a atuação do enfermeiro da UTI, quando este diagnostica distúrbios emocionais nos clientes que ali se encontram.

\section{SUPORTE TEÓRICO}

Este estudo foi desenvolvido, utilizando-se como suporte teórico: 
- Myra Estrin Levine - Teoria de Enfermagem.

O homem é considerado como um "TODO" dinâmico, onde as respostas fisiológicas ocorrem de modo a permitir a sua adaptação ao meio. Inclui em seus pressupostos, a interação cliente-enfermeiro, sendo esta influenciada pela percepção de ambos. Envolve ainda princípios de conservação de energia, onde o indivíduo, como um ser holístico, irá estabelecer reajustes orgânicos necessários à sua integridade estrutural, pessoal e social, visando a manutenção do equilíbrio adequado para ter saúde. $\mathrm{O}$ enfermeiro atua através de ações de intervenção no meio ambiente e no cliente, com o objetivo de promover a cura ou a reabilitação, estando implícita a avaliação das ações desenvolvidas.

Considera ainda a Teoria Holística que cada resposta do organismo envolve todos os recursos da pessoa, estando o todo do indivíduo em cada aspecto do ser-saúde-doença. A Teoria Holística oportuniza a maior humanização na assistência de enf ermagem.

- João Mohana - Níveis distintos da vida psíquica

A contribuição deste teórico para a enfermagem é sobretudo no que tange à denominação das necessidades que envolvem a vida psíquica do ser humano. Esta se faz em três níveis distintos e interligados:

O nível psicobiológico

O nível psicossocial

O nível psico-espiritual

Refere-se ele à existência de componentes inconscientes em cada um desses planos, que surgem como forças independentes do conhecimento, cultura, educação ou meio. Estas forças são nossos instinto's, também chamados impulsos, tendências, necessidades fundamentais. Enriquece ainda suas reflexões com considerações sobre os recursos que o indivíduo utiliza como forma de superar uma necessidade de um nível, quando buscará, através de mecanismos próprios, plena estabilização interna pela dinamização dos níveis de vida psíquica superiores ao nível onde se processou a frustração.

Evidencia-se deste modo a sua concepção do homem como ser holístico dinâmico, cuja situação de doença afetará não apenas uma necessidade humana básica, mas sim o seu todo. Neste momento, irá estabelecer reajustes orgânicos e comporta- mentais como forma de retornar à sua situação de equilíbrio.

- Abraham H. Maslow - Teoria Humanística

A inclusão deste teórico deve-se, principalmente, a importante estudo realizado sobre Motivação Humana, onde demonstra que o homem, como espécie, possui necessidades que podem ser identificadas e categorizadas para estudo. Estabeleceu ainda a hierarquia das necessidades, em que estas constituem forças motivadoras que se expressam em comportamentos, visando a constância interna e harmônica com o ambiente externo.

Considerando que existem necessidades comuns a todos os homens, MASLOW ${ }^{13}$ implementou a hierarquia de necessidades a partir do ponto de vista orgânico, que relaciona o homem e suas necessidades como uma unidade comportamental, estabelecendo um relacionamento entre as várias necessidades e as circunstâncias nas quais devem ser consideradas. Admite que, como um todo em funcionamento, o homem dá mais valor àquelas necessidades mais importantes à sua imediata sobrevivência, seguindo-se aquelas consideradas menos cruciais. Deste modo, a hierarquia das necessidades segundo MASLOW ${ }^{13}$ vai das mais críticas à sobrevivência às menos críticas.

Esquematizando-as, teríamos:

- Necessidades fisiológicas (fome, sono)

- Necessidades de segurança (estabilidade, ordem)

- Necessidades de amor e pertinência (família, amizade)

- Necessidades de estima (auto-respeito, aprovação)

- Necessidades de auto-realização (desenvolvimento de capacidades)

MASLOW ${ }^{13}$ aponta que as necessidades se arrumam elas mesmas em ordem hierárquica, onde as mais fundamentais à sobrevivência do homem devem ser prioritariamente atendidas.

Ao considerar o homem como um ser holístico, onde a situação de doença resulta em um desequilíbrio com repercussão no seu todo, MASLOW $^{13}$ refere-se a este como uma força motivadora que ativa o comportamento para aliviar a necessidade. Considera que as necessidades humanas agem de modo direcionado determinando a seleção e direção dos comportamentos que devem aliviar o desequilíbrio. Quando este é corrigido, a necessidade permanece, ainda que inativa, até que 
outro desequilíbrio ocorra. Se o comportamento regulador for eficiente, o equilíbrio pode ser mantido.

\section{METODOLOGIA}

\section{Natureza do Estudo}

Trata-se de um estudo Descritivo exploratório, ou Descritivo Survey.

\section{Local do Estudo}

O estudo foi desenvolvido em três Hospitais que tinham Unidades de Tratamento Intensivo, sendo um Hospital Universitário, um Hospital Municipal e um Hospital da Rede Previdenciária. A escolha destes procedeu-se através de sorteio.

\section{População}

A população estudada incluiu todos os enfermeiros que atuam na UTI dos hospitais escolhidos, não importando o tempo de graduação, o tempo de sua atuação no setor, a habilitação cursada, cursos de especialização ou outros relativos a UTI. A determinação da população constou de um levantamento prévio nos hospitais escolhidos do número total de enfermeiros que atuam na UTI, não importando o número total de enfermeiros de cada hospital.

\section{Instrumento para coleta de dados}

Para a realização da observação, foram elaborados três instrumentos:

1 - Formulário para observação dos sinais e sintomas mais comuns relativos à insegu. rança emocional dos clientes em UTI;

2 - Formulário para observação de como são identificados e diagnosticados pelos enfermeiros da UTI, os problemas rela. tivos à segurança emocional desses clientes;

3 - Mapa para observação sistemática não participada intensiva da atuação do enfermeiro quando este diagnostica distúrbios emocionais em clientes na UTI.

Os dados foram coletados a partir da aplicação do formulário a todos os enfermeiros que realizavam suas atividades na UTI dos hospitais selecionados.

234 - Rev. Bras. Enf., Brasília, 38(3/4), jul./dez. 1985

\section{Tratamento Estatístico}

Os resultados obtidos foram processados manualmente pelo método five cross, sendo estes em seguida tabulados e analisados a partir da determinação da freqüência absoluta e relativa. Estes dados são apresentados em tabelas através de percentual, e em quadros.

\section{APRESENTAÇÃO E INTERPRETAÇÃO DOS RESULTADOS}

Inclui-se a apresentação das tabelas resultante dos dados obtidos no instrumento denominado Mapa para Observação Sistemática não Participada Intensiva da Atuação do Enfermeiro quando este diagnostica distúrbios emocionais em clientes na UTI. Embora os três instrumentos tenham sido utilizados simultaneamente, permitindo deste modo uma melhor compreensão da atuação do enfermeiro, visto que esta é direcionada em função das necessidades sentidas e exteriorizadas pelos clientes, é nas tabelas de número 1 a 6 que se apresentam os dados relativos à observação sistemática não participada intensiva da atuação do enfermeiro na UTI, conforme verifica-se a seguir.

TABELA 1 - Classificação da comunicação utilizada pelo enfermeiro para identificar a neces. sidade de segurança emocional em clientes na UTI

\begin{tabular}{l|c|c}
\hline Tipo de comunicação & F & $\%$ \\
\hline Verbal & 23 & 53,5 \\
Não verbal & 20 & 46,5 \\
\hline Total & 43 & 100,0 \\
\hline
\end{tabular}

Nota: Os índices percentuais foram calculados com base no valor total encontrado na tabcla, uma vez que alguns enfermeiros utilizam os dois tipos de comunicação.

Verifica-se na tabela acima que das quarenta e três comunicações utilizadas pelos vinte e oito enfermeiros, vinte e três foram do tipo verbal $(53,5 \%)$ e vinte não verbal $(46,5 \%)$, sendo que alguns enfermeiros utilizam os dois tipos de comunicação.

Verifica-se na Tabela 2 que o elemento mais influente na percepção do enfermeiro para identificar e diagnosticar problemas emocionais é o conhecimento com o percentual igual a $19,8 \%$, seguido da intuição com $17,6 \%$. Os demais expressam indices semelhantes. 
TABELA 2 - Elementos que influenciam a percepção do enfermeiro na identificação e diagnóstico de problemas emocionais em clientes na UTI

\begin{tabular}{l|c|c}
\hline $\begin{array}{l}\text { Elementos que influenciam } \\
\text { a percepção }\end{array}$ & F & $\%$ \\
\hline Vivências & 21 & 16,0 \\
Valores éticos & 20 & 15,3 \\
Valores pessoais & 22 & 16,8 \\
Sentimentos & 10 & 7,6 \\
Reflexão & 9 & 6,9 \\
Conhecimentos & 26 & 19,8 \\
Intuição & 23 & 17,6 \\
\hline Total & 131 & 100,0 \\
\hline
\end{tabular}

Nota: Os índices percentuais foram calculados com base no valor total encontrado na tabela, uma vez que a percepção do enfermeiro inclui mais de um elemento, sendo estas variáveis conforme a situação.

TABELA 3 - Tipo de observação utilizada pelo enfermeiro na UTI para diagnosticar problemas emocionais nos clientes

\begin{tabular}{l|c|c}
\hline Tipos de Observação & F & $\%$ \\
\hline Direta (Experiência) & 17 & 11,6 \\
Indireta: - Comentários & 28 & 18,9 \\
$\quad$ - Relatórios & 28 & 18,9 \\
- Discussões & 23 & 15,5 \\
- Visitas & 25 & 16,9 \\
$\quad$ - Anotações & 27 & 18,2 \\
\hline Total & 142 & 100,0 \\
\hline
\end{tabular}

Nota: Os índices percentuais foram calculados com base no valor total encontrado na tabela, uma vez que alguns enfermeiros utilizam os dois tipos de observação sendo estas, na maioria das vezes, apoiadas em mais de uma fonte.

Verifica-se nesta tabela que a observação indireta é a mais utilizada pelos enfermeiros na UTI. Com índices mais expressivos encontram-se os comentários e relatórios com valor percentual igual a $18,9 \%$, seguidas de anotações com $18,2 \%$. As discussões e visitas apresentaram índices de 15,5\% e $16,9 \%$, respectivamente. O menor percentual foi registrado na observação direta, ou seja, 11,6\%.

Verifica-se na tabela 4 que a atitude mais constante dos enfermeiros ao diagnosticarem problemas emocionais em clientes na UTI é a ajuda, com índice percentual de $13,6 \%$, seguindo-se a benevolência e respeito com o mesmo percentual, ou seja, 12,9\%. Interesse, compreensão, simpatia e empatia registraram índices expressivos, enquanto que aborrecimento e irritação registraram os menores índices percentuais $(1,8 \%)$. Não foram observadas as atitudes de antipatia, preocupação ou zanga.

TABELA 4 - Atitudes dos enfermeiros quando estes diagnosticam problemas emocionais em clientes na UTI

\begin{tabular}{l|r|c}
\hline Atitudes dos enfermeiros & F & $\%$ \\
\hline Solidariedade & 20 & $12, \overline{5}$ \\
Ajuda & 22 & 13,6 \\
Benevolência & 21 & 12,9 \\
Respeito & 21 & 12,9 \\
Aborrecimento & 3 & 1,8 \\
Interesse & 17 & 10,4 \\
Antipatia & - & - \\
Empatia & 14 & 8,6 \\
Tolerância & 11 & 6,7 \\
Compreensão & 16 & 9,8 \\
Irritação & 3 & 1,8 \\
Preocupação & - & - \\
Simpatia & 15 & 9,2 \\
Zanga & - & - \\
\hline Total & 163 & $100,0 \%$ \\
\hline
\end{tabular}

Nota: Os índices percentuais foram calculados com base no valor total encontrado na tabela, uma vez que alguns enfermeiros apresentam quase simultaneamente mais de uma atitude, sendo estas variáveis dependentes da situação em que se encontravam.

TABELA 5 - Tipos de intervenção utilizada pelos enfermeiros quando diagnosticam problemas emocionais nos clientes da UTI

\begin{tabular}{l|c|c}
\hline Tipos de Intervenção & F & $\%$ \\
\hline Ambiental & 27 & 49 \\
Cliente & 28 & 51 \\
\hline Total & 55 & 100 \\
\hline
\end{tabular}

Nota: Os índices percentuais foram calculados com base no valor total encontrado na tabela, uma vez que a maioria dos enfermeiros intervém simultaneamen te no ambiente e no cliente.

Evidencia a tabela 5 que a intervenção mais utilizada pelos enfermeiros, uma vez diagnosticados problemas emocionais nos clientes da UTI, é a realizada no cliente $(51 \%)$, embora a diferença de percentual relativa ao ambiente seja mínima levando-se em consideração que tal índice é de $49 \%$, donde se deduz que vinte e oito enfermeiros intervêm concomitantemente no cliente e ambiente, $e$ 
apenas uns nâo realizam a intervenção no ambiente.

TABELA 6 - Ações realizadas pelo enfermeiro quando este diagnostica problemas emocionais em clientes internados na UTI

\begin{tabular}{l|r|r}
\hline Ações do Enfermeiro & F & $\%$ \\
\hline 1. Não verbais & & \\
- Modo de olhar & 25 & 15,7 \\
- Tom de voz* & 26 & 16,4 \\
- Toca o cliente & 25 & 15,7 \\
- Segura o equipamento & - & - \\
- Apenas escuta & 2 & 1,2 \\
- Outros & - & - \\
2. Verbais & 23 & 82,1 \\
- Conversa & 26 & 92,6 \\
- Orienta & 18 & 64,3 \\
- Aconselha & 14 & 50,0 \\
- Procura distraí-lo & 159 & 100,0 \\
\hline Total & \\
\hline
\end{tabular}

Nota: Os índices percentuais foram calculados com base no valor total encontrado na tabcla, uma vez que a maioria dos enfermeiros utiliza ações verbais e não, simultaneamente.

* Emissão de sons não diretamente relacionados com o cliente.

Entre as ações verbais e não verbais realizadas pelo enfermeiro, a mais freqüente entre as verbais é a orientação $(92,6 \%)$, seguida da conversa $(82,1 \%)$.

Apenas $50 \%$ dos enfermeiros procuram distrair o cliente quando diagnosticam problemas emocionais. $\mathrm{O}$ maior índice percentual no item 1 , relativo a ações não verbais, foi encon trado no tom de voz - 16,4\%, sendo este entendido como a emissão de sons não diretamente relacionados ao cliente, incluindo conversas sobre variados assuntos, comentários sobre a situação do cliente e outros. O modo de olhar e o tocar no cliente expressaram o mesmo percentual, sendo este de $15,7 \%$. 0 ouvir é utilizado apenas por dois enfermeiros $(1,2 \%)$.

\section{CONCLUSÕES}

A partir do estudo realizado pode-se concluir que:

- o atendimento às necessidades psicobiológicas é priorizado pelos enfermeiros que atuam nas UTI observadas, em detrimento das necessidades psicossociais e psico-espirituais;

- a carência de enfermeiros nos quadros da equipe de enfermagem das UTIs pesquisadas compromete seriamente o atendimento às necessidades psicobiológicas dos clientes ali internados, sendo este comprometimento maior ao considerar-se o atendimento às necessidades psicossociais e psico-espirituais;

- o cenário institucional, capaz de influenciar a assistência prestada, vem alterando o significado real da relação de ajuda, por delegar aos enfermeiros tarefas ou atribuições que os incompatibilizam com seu próprio papel social, visto que o enfermeiro é um elemento importante na equipe multiprofissional de saúde, principalmente por ser responsável pela qualidade da assistêńcia prestada;

- o nível de comunicação do enfermeiro com o cliente é prejudicado devido à utilização de uma linguagem rebuscada; inacessível ao nível de compreensão dos clientes;

- os enfermeiros da UTI tendem a afastar-se do cuidado a clientes com problemas emocionais, devido ao receio de envolverem-se em conflitos;

- os problemas emocionais são, em sua maioria, diagnosticados pelo enfermeiro através de observação indireta, mais precisamente a partir de comentários e relatórios;

- o enfermeiro utiliza seus conhecimentos, valores e sentimentos na identificação e diagnóstico dos problemas emocionais, e não inclui a análise e reflexão em seus procedimentos, o que acarreta a eleição de ações quase sempre ineficazes ao problema do cliente;

- apesar de estarem cientes dos distúrbios emocionais nos clientes da UTI, os enfermeiros expressam dificuldades que abrangem desde a identificação e diagnóstico, até a adoção de atitudes e ações adequadas à resolução de problemas.

\section{RECOMENDAÇÃO}

Urge que os enfermeiros repensem, analisem e discutam os aspectos relacionados à sua prática, sob pena desta ser absorvida por outros profissio- 
nais, incorrendo portanto no risco de extinguir-se como profissão no contexto social.

ESPIRITO SANTO, T. J. M. Emotional assurance how basic human need: nurse care to the client in the Intensive Unit Therapy. Rev. Bras. Enf., Brasília, 38 (3/4): 231-237, July/Dec. 1985.

\section{REFERÊNCIAS BIBLIOGRAFICAS}

1. BRASIL. Ministério da Saúde. Secretaria Nacional de Ações Básicas de Saúde. Normas e padrōes de construção e instalaçōes de Serviços de Saúde. Brasília, DNOSS, 1979.

2. CARNEIRO, A. Comunicação enfermeiro-paciente na unidade de terapia intensiva - essencial à boa assistência de enfermagem. Rio de Janciro, 1982. 147 p. Tese (mestrado) - UFRJ. Escola de Enfermagem Ana Neri.

3. CARVALHO, V. A relação de ajuda e a totalidade da política de enfermagem. In: CONGRESSO BRASILEIRO DE ENFERMAGEM, 32. Brasília, 1 a 7 de junho de 1980. Anais... Brasília, ABEn, 1980. p. 65-72.

4. COSTA, L. A. T. Situaçōes VIDA MORTE participacão do enfermeiro. Rio de Janeiro, 1977. 98 p. Tese (mestrado) - UFRJ. Escola de Enfermagem Ana Neri.

5. DABOIT, A. A. \& VAS, N. F. Assistência de enfermagem junto às variaçōes comportamentais do paciente em clínica geral. Rev. Bras. Enf., Brasília, 31 (4): 443-8, out./dez. 1978.

6. DANIEL, F. Atitudes interpessoais em enfermagem. São Paulo, EPU, 1983. 185p.

7. EPSTEIN, C. Interação efetiva na enfermagem. São Paulo, EPU/EDUSP, 1977. 173 p.

8. GEORGE, J. B. \& LEVINE, M. E. Nursing theories - the bases for professional nursing practice. New Jersey, Pren tice Hall, 1980. p. 150-63.

9. GONÇALVES, M. M. G. Enfermagem e segurança emocional do paciente, Rev. Enf. Novas Dimens., São Paulo, 5 (1): 31-6, jan./f ev. 1979.
10. HORTA, W. Processo de enfermagem. São Paulo, EPU/EDUSP, 1979.

11. KAMADA, C. et alii. Equipe multiprof issional em unidade de terapia intensiva. Rev. Bras. Enf., Brasília, 31 (1): 60-7, jan./mar. 1978 .

12. L.EITE, J. L. Assistência de enfermagem em cirurgia cardiaca - alteraçōes de comportamento no pósoperatório. Rio de Janeiro, 1975. 68 p. Tese (Livre Docência) - FEFIEG. Escola de Enfermagem Alfredo Pinto.

13. MASLOW, A. H. Introdução à psicologia do ser. 2. ed. Rio de Janeiro, Eldorado Tijuca, s.d.

14. MOHANA, J. M. O mundo e eu. 7. ed. Rio de Janeiro, Agir, 1978. 248 p.

15. NÓBREGA, M. R. S. Identificaçãu de problemas do cliente, que podem ser assistidos pela enfermeira. In: CONGRESSO BRASILEIRO DE ENFERMAGEM, 29., Camboriú, outubro, 1977. Anais... Camboriú, ABEN, 1977. p. 39-50.

16. ORGANIZAÇAO PANAMERICANA DA SAÚDE. Plan decenal de salud para las Américas; informe final de la 3. Reunión Especial de ministros de salud de las Américas. Washington, 1973. (Documento of icial, 118).

17. ORLANDO, I. J. O relacionamento dinâmico enfermeiro/paciente. São Paulo, EPU/EDUSP, 1975.

18. PAIM, L. Algumas considerações de enfermagem sobre as necessidades psicossociais e psico-espirituais dos pacientes. Rev. Bras. Enf., Brasília, 32 (2):160-6, abr./jun. 1979.

19. SELYE, H. The stress of life. New York, Book Company, 1956.

20. SILVA, T. C. Enfermagem e atenção psico-espiritual à pessoa humana. Rio de Janeiro, 1979. $103 \mathrm{p}$. Tese (mestrado) - UFRJ. Escola de Enf crmagem Ana Neri.

21. SIMĀO, A. T. Centro de tratamento intensivo. In: Terapia intensiva. Rio de Janeiro, A theneu, 1976. p. 7-23.

22. TROTTO, G. et alii. O ser humano no centro de tratamento intensivo. In: SIMĀO, A. T. Terapia intensiva. Rio de Janeiro, A theneu, 1976. p. 607-24. 